\title{
Effective Traffic Management Technique to Clear Roads for Emergency Services
}

\author{
Sanam Kazi, Shaikh lqra, Shaikh Kausar, Sheikh Azim, Modak Muaz \\ M.H SabooSiddik College of Engineering, Byculla \\ Mumbai 400008
}

\begin{abstract}
Road congestion results in sizably voluminous waste of time of millions of people. The emergency accommodations like ambulance and fire brigade has to face a tough time because of road congestion. This can additionally have numbers of effects on drivers, health and environment. The possible way to deal with such quandary is to have a distributed system that gives the best route to the driver and avail the traffic ascendant entities to clear the road that can avail these emergency accommodations reach the destination in less time. For this reason, in this paper, we are giving an archetype that can avail the emergency accommodations. In this, we have designed a system to solve the quandary of traffic congestion for ambulance to reach its destination without wasting time in traffics. To ken about the traffic situations and how this quandary is initially been done a survey was implemented. This study is predicated on the traffic survey data from Mumbai and our system betoken that such system can be implemented which indeed can ameliorate the traffic flow for emergency accommodations.
\end{abstract}

\section{General Terms}

Road congestion, Ambulance, Communication, Vehicles, Signals.

\section{Keywords}

GPS, Emergency services, Traffic congestion, Mobile Application.

\section{INTRODUCTION}

The major cause leading to traffic congestion is the high number of conveyance which was caused by the population and the development of economy. Every day four thousand conveyances are registered and come on road. Traffic congestion can have a number of effects on drivers, the environment, health and the economy. Millions of people deal with traffic congestion on a quotidian substructure additionally the emergency accommodation like Ambulance and Fire Brigade face the same issue which leads in a delay to reach the terminal.

\subsection{Motivation}

The delay in ambulance services due to traffic jam has lead in taking lives of many humans. There are many cases in India that caused death just because of road congestion.Delay in reaching the hospital caused the death of a newborn after an ambulance carrying the baby allegedly got stuck in a traffic jam because of a cycle rally. The child died soon after reaching PGIMS at Rohtak as the ambulance was stuck for "20 to 30 minutes" due to the cycle rally[1].There are many such cases that caused death due to thedelay of services. The 'Sanjeevni

108' ambulance servicestarted by the centre to provide immediate help to patients in emergency and those injured in road mishaps, face this serious issue of traffic jams which often puts patients' life at peril in the city. As per the 'Golden Hour' theory a patient of road mishap has to be brought to a hospital within an hour, which increases chances of survival. The 'Golden Hour' theory has been framed by WHO (World Health Organization), which is followed all over the world. As per the said theory if a patient of road mishap or a heart patient is rushed to a hospital within an hour,his chances of survival go up by 70 to 80 per cent.According to facts, during the last six months 1200 persons of the district have been brought to hospitals in 'Sanjeevni 108' ambulances, out of which 12 have died in ambulances because of getting stuck in a traffic jam in the city. Apart from patients of road mishaps, heart patients are also needed to be rushed to hospitals in time. However, due to traffic jams and people not bothering to give way to Ambulances, many such patients also have lost their lives before reaching a hospital [4]. According to the National Crime Records Bureau, nearly 24,012 people die each day due to a delay in getting medical assistance. These patients have suffered heart attacks, brain haemorrhage, suicide attempts, accidents and strokes.According to the National Rural Health Mission (NRHM), 6,226 ambulances are available with the government to cater to the country's rural areas. This is half the required number of ambulances [2]. As per the WHO standards, there should be at least one ambulance per 100,000 population in the plains, and one for every 70,000 population in hilly terrain or tribal areas where population is scattered.The goal of an ambulance is to reach any place within 15-20 minutes after the distress call and transport the patient to a health facility within 20 minutes thereafter.Every second heart attack patient in India takes more than 400 minutes to reach a hospital, which is almost 13 times more than the ideal window of 30 minutes, government data shows. A two-year data from the ongoing Management of Acute Coronary Event (MACE) Registry of the Indian Council of Medical Research (ICMR) shows at some places it even takes 900 minutes as a lot of time is wasted in transportation. A lot of precious time is still being wasted in taking a heart attack patient to a facility that is capable of treating heart attacks. The delay mostly is because of the patient being remotely located in rural areas or belonging to a terrain that is difficult to access.

Hence this paper proposes a solution to solve this quandary of delay for emergency accommodations due to traffic congestion. It aims to cognizant the signals about an emergency accommodation or conveyance is approaching towards them so it will glow in different way so that traffic police and the people on the road will get a prior notice about it and the will commence making a way for that respective conveyance. It will give a prior cognizance to the signal regarding the emergency accommodation arriving. Our main motive to make the signals vigilant about the Emergency Accommodation passing by, so that the conveyances and the 
traffic police can make a way for the emergency accommodation so that they can be on time or afore time to preserve some precious life around us.

\section{EXISTING SYSTEM AND ITS DRAWBACK}

To find the solution a survey was carried out toaccumulatedata from the traffic officers. This survey was done in different locations of the city to ken more about the traffic scenarios in the city. During this survey additionally, the ascertained subsisting system that currently the traffic ascendant entities are utilizing to avail the road congestion. Initially the traffic officers utilize the walkie talkie to communicate so to clear the roads. But as every silver cloud has its gray lining the utilization of subsisting system withal have some drawbacks.

1. In some areas they have to apportion a channel with several other users. Categorically in dense urban areas, radio channels can become congested with multiple users and exclusivity can become arduous to obtain.

2. The advertised range of the units should be under perfect conditions-dry air, direct line of sight, no vegetation blocking the signal.. Your best results may only be a tenth of the advertised range if there is atmospheric interference, abundant vegetation, or high humidity.

3. Even if you're within your working range for the terrain, if a ridge or hill blocks your line of visual perception, it can and probably will block the transmission.

4. The walkie-talkie work on batteries so this is a conflict that the batteries can go dead.

\section{PROPOSED SYSTEM}

\subsection{Introducing new novel symbol for efficient management}

This approach fundamentally aims at displaying a symbol in the already subsisting signal light. In Red traffic signal light an ambulance symbol will be displayed i.e. plus symbol that will blink perpetually in the signal when the emergency conveyance is 250 meters away.

An ambulance accommodation siren is auricularly discerned from an average distance of 90 meters[7]which is too less for an traffic police to clear the roads.

So in lieu of making the traffic police cognizant at the last minute we can make them vigilant about the arriving emergency accommodation when it is 250 meters away from the traffic signal.

The symbol will commence blinking in the red traffic light that will make the traffic police immediately cognizant about the emergency arriving and hence will make the task facile for them to clear the roads as anon as possible.

The minimum distance between the two traffic signals is 800 meters (i.e 2640feets)so there is a lot of time required for an emergency vehicle to travel between two traffic signals.So our solution will simply give an prior knowledge to the traffic police at particular signal that an emergency vehicle is arriving and is at the distance of 250 meters away from traffic signal.

This approach will be using the microprocessor software and the concept of programming LED'S to blink the symbol in traffic signal light. The ambulance driver will be using the Mobile application to find the best route for its destination and after selecting the route the lights only on that particular route willblink when the emergency vehicle is 250 meters away from that signal. This will be implemented using the GPS (Global Positioning System) that will help the driver to find best route and only then blink the LED's.

So this approach will just add a new symbol of ambulance in the existing traffic signal light of Red colour that will blink and hence there won't be any hardware installation or requirement for new setup and only the software addition will be required.

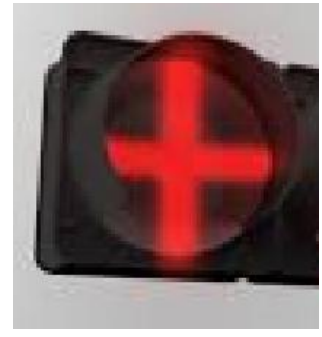

Figure 1: Introducing new symbol

\subsection{Introducing a new colour light in the traffic signal}

This approach will basically aim in installing a new hardware that will be installing a new colour light in the Traffic signals. This will include installation of new traffic signal with BLUE colour light that will indicate that an emergency service is arriving. First that will be known only to the traffic police that blue light indicates that an ambulance is arriving but in no time all the people will be aware about that and can willingly give a side to the emergency service so that it reaches in time.[3] So in this approach we need to install either a completely new signal or install a new light in the signal. Since completely new installation will be too much cost consuming we can add a new light in existing signals. This will be more effective since blue light will be easily noticeable and hence will give more effect on the signal.Very soon everyone will become aware about the new light and it's purpose.

This will help the traffic police in clearing the road since siren is not that audible from a far distance.

As this will give a prior indication about the emergency service arriving it will be easy for traffic police as well as emergency service driver to make a way out of the traffic.

Blue colour will be easily visible and will have its own significance. Since Mumbai faces a huge traffic issues and there are approximately 600 traffic signals overall in Mumbai so this won't lead in much time consuming and cost consuming for installing new lights at 600 signals[5].

A new hardware will also lead in a new start since we have been using the old signals from so long time. There are many issues occurring and the maintenance leads in huge cost consumption so instead of recovering it everytime a new installation will solve the issue. And also at many places there are older versions of signals that haven't been replaced by new technology signals so this problem can also be solved by new installation.

So we came up with the second approach of installing all new signal that will change all the older versions of traffic signals 
and replace it with new ones. This approach will also setup a new standard of signals and will be very helpful for the emergency services so that they can reach their destination place as soon as possible.

It will give a prior knowledge to the signal regarding the emergency service arriving. This approach will basically aim to make the signals aware about the Emergency Service passing, by using a new colour in traffic signals so that the vehicles and the traffic police can make an easy way for the emergency service to reach the destination faster.

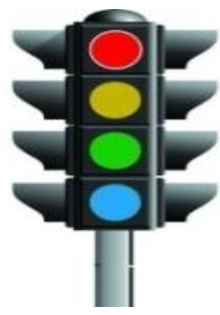

Figure 2:Installation of new colour light $[8]$

\section{BLOCK DIAGRAM}

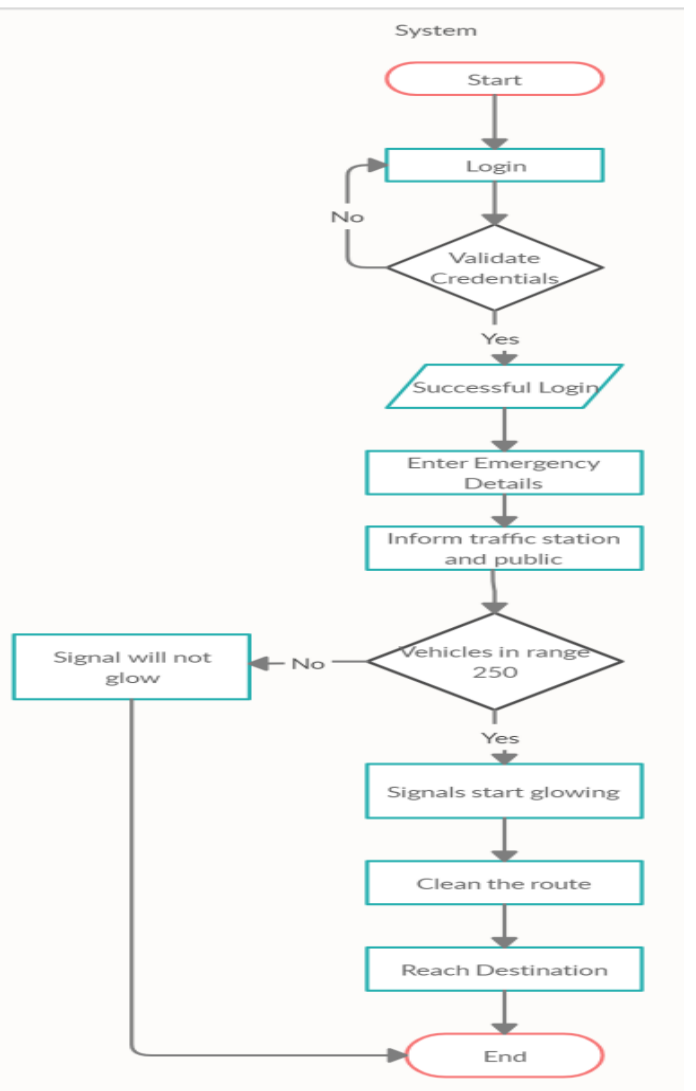

Figure 3:Block Diagram for system

\section{METHODOLOGY:}

The traffic congestion has a sizably voluminous impact on the emergency accommodations, millions of people are dealing with it everyday. The traffic ascendant entities endeavor their best to avail the emergency accommodations but due to the incrementation population and conveyances it is becoming arduous and an abundance of time is wasted in the traffic for the accommodations.[6]

To resolve this issue a survey was conducted to amass information about the traffic congestion in the city. This survey was conducted for the traffic officers as what quandaries they face in clearing roads for an emergency accommodation.

The survey took place in different regions of the city and the officers to ken about the different traffic situations across the city. This survey availed us in finding patterns and categorizing .

To get more information avisit tothe Mumbai Traffic Control Head Quarters in worli, the API officer Mr.SandipGovindFanse who gave us detailed information about the signals in Mumbai. He disclosed about how they manage sizably voluminous traffic especially when an emergency accommodation has to pass. A questionnaire was yare to interrogate the traffic officers to amass data.

The accumulated data was then analysedto find different patterns and statistics around the city.

\subsection{Algorithm}

1) The system commences with the mobile application where the utilizer has to authenticate to the system and integrate the details of the destination place they have to reach.

2) After successful authenticate the utilizer is redirected to the main page where the utilizer has to enter the destination details else the utilizer is asked to authenticate correctly.

3) Once the utilizer enters the all emergency details and the location the system culls the most proximate and shortest path for the utilizer to peregrinate in as less time as possible.

4) Once the utilizer accepts the route and follows the Map the signals on that particular path gets activated.

5) When the utilizer starts travelling on that particular route and is $250 \mathrm{~m}$ away from the signal, the signal will automatically start glowing the emergency accommodation light denoting that the emergency accommodation is arriving.

6) The traffic police and people at that signal will get notified and immediately endeavour to make a way for the emergency conveyance.

7) This signal will stop glowing as anon as the emergency conveyance passes the signal and the next signal will get activated when the emergency conveyance is at a distance of $250 \mathrm{~m}$ away.

8) And hence the Emergency conveyance will be able to reach the destination place as anon as possible without delay due to traffic.

\section{RESULTS AND ANALYSIS}

Following is the result analysis that was discovered from the survey. Figure4 represents how much time the traffic police requires to clear the road, division wise according to our survey. It has been found in the survey that Malad division requires more time in order to clear the road for emergency services, whereas Bandra division takes less time to clear the traffic. The reason why malad takes more time to clear is because the roads are very compact also the vehicles crowd is more which results in heavy traffic,the population is also one of the reason resulting in huge traffic hence malad division takes more time to clear traffic. Hence the average time required to clear traffic is 48 seconds. 


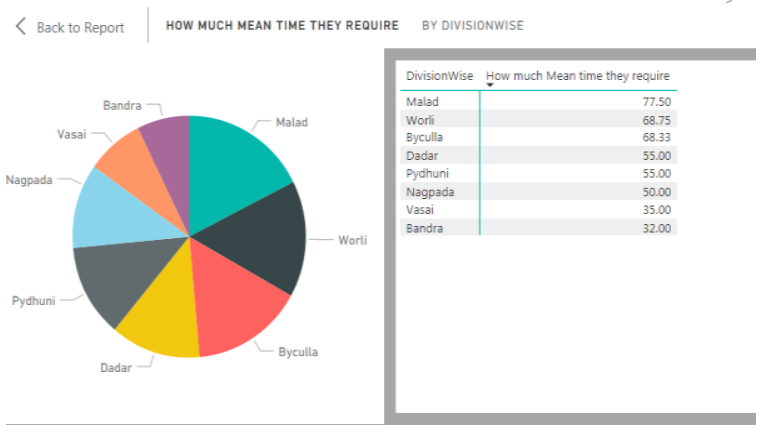

Figure4:Mean time required

The following pie chart display[Figure 5] how many people co-operate with the traffic police to clear the road, division wise. In bandra division more number of people cooperate with the traffic police whereas in colaba division very less people cooperate. In bandra the roads are wide which also results in less traffic the people here are also cooperate which helps the traffic police to clear road very easily.

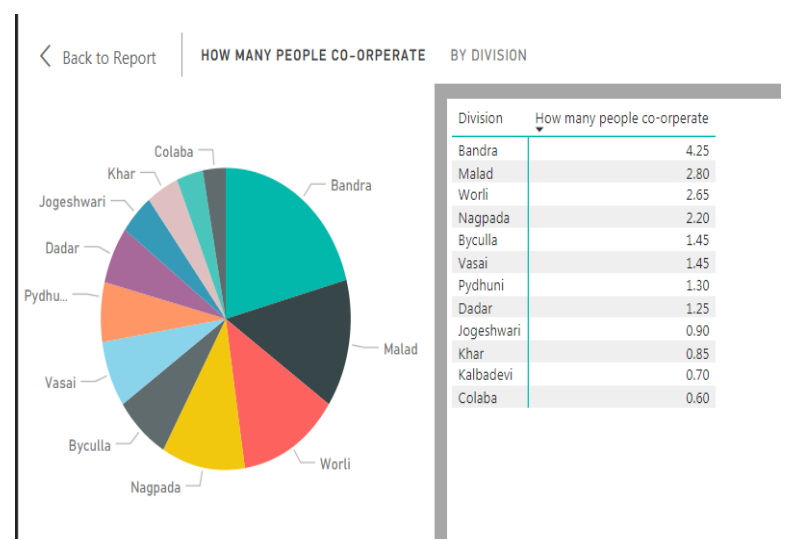

Figure5:Mean of people that cooperate

According to the survey Figure 6 represents how many people likes the solution based on ranks i.e. very good,excellent and good. So many people ranked it as very good saying that the solution can help them in many ways as they can get a prior knowledge about the emergency services which can help themclear roads on prior notice.
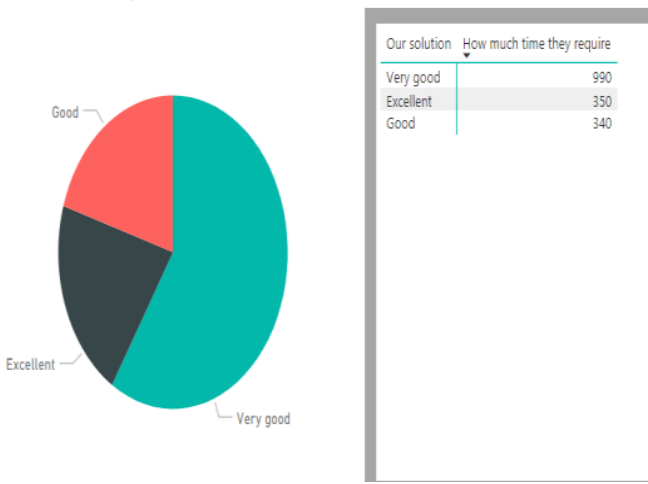

Figure 6: Likes on our solution

Also in Figure 7 it is shown how much the solution can help the traffic police if they get a prior notice about an emergency service passing by the road. Majority of traffic police said yes as it can be very helpful for them because sometimes it's difficult to communicate on a walkie talkie therefore this method can make it more easy for us to clear roads

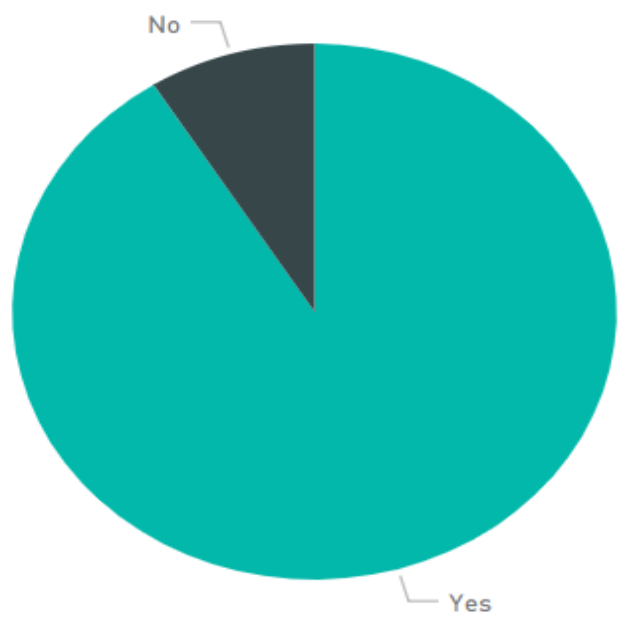

Figure 7:How much this solution can help them

Figure 8 represents how traffic police get to know about the emergency services. There are two ways either by siren or walkie talkie. Division wise it was found that in most of the division they get to know with the help of siren. Walkie talkie also helps them in some cases otherwise with siren they get to know about the emergency services.

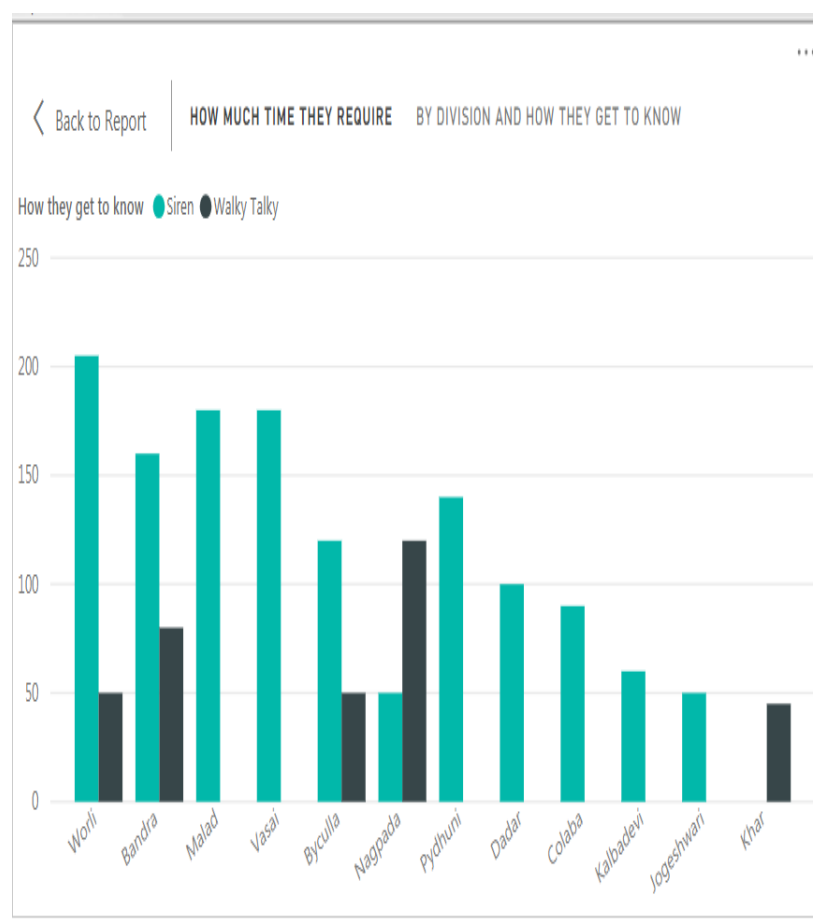

Figure 8: How traffic police gets to know about Emergency Vehicle

A huge dataset was collected from the survey in different divisions to know about the working of traffic signals and study the routine of emergency vehicles. Figure 9 is the screenshot of dataset collected during the survey. 
Following is the data that we collected from different areas:

\begin{tabular}{|c|c|c|c|c|c|c|}
\hline 1 & Name & Division & How they get to know & How much time they require & How many people co-orperate Prior notice & Our solution \\
\hline 2 & Pradeep shinde & Bandra & Walky Talky & 30 & $75.00 \%$ Yes & Excellent \\
\hline 3 & Abhijeet Govalkar & Bandra & Walky talky & 25 & $70.00 \%$ Yes & Very good \\
\hline 4 & Kamble Ashok & Bandra & Siren & 40 & $60.00 \%$ Yes & Very good \\
\hline 5 & Patel Dhunji & Bandra & Siren & 40 & $70.00 \%$ No & Very good \\
\hline 6 & Kiran Landge & Bandra & Walky talky & 25 & $80.00 \%$ Yes & Very good \\
\hline 7 & Anna Sathe & Bandra & Siren & 90 & $75.00 \%$ Yes & Very good \\
\hline 8 & Narayan Aapte & Byculla & Siren & 35 & $60.00 \%$ Yes & Very good \\
\hline 9 & Sandip Govind & Byculla & Siren & 80 & $50.00 \%$ Yes & Very good \\
\hline 10 & Satish altekar & Colaba & Walky talky & 60 & $80.00 \%$ No & Good \\
\hline 11 & Sampat Varpe & Dadar & Siren & 50 & $70.00 \%$ Yes & Excellent \\
\hline 12 & Anant Altekar & Dadar & Siren & 90 & $60.00 \%$ yes & Good \\
\hline 13 & Sunil Soni & Jogeshwari & Siren & 60 & $70.00 \%$ yes & Excellent \\
\hline 14 & Sopan Nighot & Kalbadevi & Siren & 80 & $60.00 \%$ yes & Very good \\
\hline 15 & Shilpa Ghure & Khar & Siren & 60 & $70.00 \%$ yes & Very good \\
\hline 16 & Kailash Kare & Malad & Walky talky & 60 & $70.00 \%$ yes & Good \\
\hline 17 & Gopal Agarkar & Malad & Walky talky & 50 & $80.00 \%$ yes & Very good \\
\hline 18 & Suryakant Nokulka & a Malad & Siren & 120 & $80.00 \%$ yes & Very good \\
\hline 19 & S.B. Paura & Malad & Siren & 80 & $70.00 \%$ yes & Good \\
\hline
\end{tabular}

Figure 9: Glimpse of Data collected from survey

\section{CONCLUSION}

The novel algorithm which the paper proposes summarises our research and survey over the road congestion across various regions of the city. A survey was conducted which helped in finding the scenarios and notified us on how traffic congestion is such an issue. This survey encouraged us to establish a new algorithm that will help in clearing the traffic and reduce congestion. Every day the police officers face various issues in clearing roads, the crowd is enormous and thus leads in traffic congestion. After every 15 to 20 minutes an ambulance passes which therefore becomes a task for the traffic officers to clear in less time. Thus to resolve this two solutions are proposed which will be effective and can improve the road congestion for the services in less time.

\section{REFERENCES}

[1] "NDTV," 24 August 2018. [Online]. Available: https://www.ndtv.com/india-news/delay-in-reachinghospital-caused-haryana-newborns-death-says-probereport-1905809.

[2] "Hindustan Times," 10 March 2017. [Online]. Available: https://www.hindustantimes.com/india-news/more-than50-of-heart-attack-cases-reach-hospital-late-govt-datashows/story-penFdsewgGwpIwiQnRDoLJ.html.

[3] "Mail Online," [Online]. Available: https://www.dailymail.co.uk/health/article55521/Thousands-die-ambulance-delays.html.

[4] "Times of India," 18 October 2016. [Online]. Available: https://timesofindia.indiatimes.com/city/raipur/Chokinglife-12-on-way-to-hospitals-die-in-trafficjams/articleshow/54910538.cms.
[5] "The World Bank," 15 June 2011. [Online]. Available: https://www.worldbank.org/en/news/feature/2011/06/14/ mumbai-modern-traffic-management-system.

[6] "GKToday," 14 September 2011. [Online]. Available: https://www.gktoday.in/news-today/india-roadscongress-irc/.

[7] "Physics Forums," [Online]. Available: https://www.physicsforums.com/threads/speed-of-soundvs-distance. 265240.

[8] [Online].Available:https://images.app.goo.gl/i6749E3Y6x wuxBp67.

[9] S. Djahel, M. Salehie, I. Tal and P. Jamshidi, "Adaptive Traffic Management for Secure and Efficient Emergency Services in Smart Cities," p. 4, 2013.

[10] J. G. J. S.-S. Ramon Bauza, "Road Traffic Congestion Detection through Cooperative Vehicle to-Vehicle Communications," IEEE Local Computer Network Conference, p. 7, 2010.

[11] I. Leontiadis, G. Marfia, D. Mack, G. Pau, C. Mascolo and M. Gerla, "On the Effectiveness of an Opportunistic Traffic Management System for Vehicular Networks," IEEE Transactions on Intelligent Transportation Systems, vol. 12, no. 4, p. 12, 2011.

[12] S. H. a. V. G. Rajeshwari Sundar, "mplementing Intelligent Traffic Control System for Congestion Control, Ambulance Clearance, and Stolen Vehicle Detection," IEEE Sensors Journal, vol. 15, no. 2, 2015. 